\title{
AMBIÇÃO ESTRATÉGICA DE MARKETING: O CASO DO SEGMENTO QUÍMICO-FARMACÊUTICO. ${ }^{1}$
}

\author{
MARKETING STRATEGIC AMBITION: THE CASE OF CHEMICAL- \\ PHARMACEUTICAL SEGMENT.
}

\author{
Evandro Luiz Lopes ${ }^{2}$ \\ Sérgio Luiz do Amaral Moretti ${ }^{3}$ \\ Emerson Roberto Lopes ${ }^{4}$
}

\begin{abstract}
RESUMO: O trabalho analisou as relações entre investimento no marketing mix e market share de empresas do segmento químico-farmacêutico. Esta iniciativa tenta preencher uma lacuna dos estudos de marketing metrics no país por meio da utilização parcial do conceito de ambição estratégica de marketing (Cook Junior, 1983, 1985). A metodologia foi de natureza quantitativa, transversal e exploratória e, utilizou uma escala para mensuração das variáveis do marketing mix em 4 dimensões distintas. Das 600 empresas convidadas a participar da pesquisa 114 foram consideradas. Os grupos formados na análise fatorial exploratória foram tratados como variáveis independentes e o market share como variável dependente do modelo de regressão linear multivariada. Os resultados mostraram um coeficiente de determinação $\mathrm{r}^{2}=0,206$ indicando que as variáveis do marketing mix, explicam 20,6\% da variação do market share das empresas participantes. Houve também a confirmação da correlação positiva entre investimento no marketing mix e o market share da organização indicando indícios de que a relação pode servir como indicador de ambição estratégica de marketing.
\end{abstract}

PALAVRAS-CHAVE: Ambição Estratégica de Marketing; Marketing Metrics; Marketing Mix; Market Share.

ABSTRACT: The study analyzed the relationship between investment in the marketing mix and market share of companies in the chemical-pharmaceutical sector. This initiative attempts to fill a gap in studies of marketing metrics in the country through the partial use of the concept of strategic marketing ambition (Cook Junior, 1983, 1985). The methodology was quantitative, cross-sectional and exploratory and used a scale to measure the variables of the marketing mix in 4 different sizes. Of the 600 companies invited to participate, 114 were considered. The groups formed in the exploratory factor analysis were treated as independent variables and market share as the dependent variable in linear regression analysis. The results showed a coefficient of determination $r 2=0.206$ indicating that the variables of the marketing mix, explain $20.6 \%$ of the variation of the market share of participating companies. There was also confirmation of the positive correlation between investment in the marketing mix and market share of the organization indicating that the relation could serv as indicator of marketing strategic ambition.

KEY-WORDS: Marketing Strategic Ambition; Marketing Metrics; Marketing Mix; Market Share.

\footnotetext{
${ }^{1}$ Artigo Recebido em 03.01.2010. Revisado por pares em 25.11.2010. Recomendado em 28.04.2011 por Leomar dos Santos Editor. Publicado em 28.07.2011.

Organização Responsável pelo periódico: Universidade regional de Blumenau - FURB - www.furb.br/rn
}

\footnotetext{
${ }^{2}$ Universidade Nove de Julho - UNINOVE - elldijo@uol.com.br

${ }^{3}$ Universidade Nove de Julho - UNINOVE - moretti@uninove.br

${ }^{4}$ Centro Universitário da FEI - lopeser@terra.com.br
} 


\section{INTRODUÇÃO}

O desempenho da empresa relacionado às atividades de Marketing sempre foi alvo de interesse pelas empresas, devido à dificuldade de estabelecer claramente a qual esforço específico se deve creditar o resultado alcançado. O modelo do marketing mix embora trate de forma conjunta o esforço de marketing necessita ser entendido isoladamente em seus componentes pelas ligações que cada uma estabelece com outras áreas da organização. Um bom exemplo desta situação é que os inputs da análise de portfólio das empresas, utilizados popularmente pela Matriz BCG são de fato os outputs da estratégia de marketing (COOK JUNIOR, 1983, p. 3). Assim a pergunta essencial que se colocava para a academia era (de fato, ainda é): em que esforço de produto, promoção distribuição ou comunicação depende o market share de determinado produto ou serviço?

A partir da década de 1970 esses elementos foram trabalhados mais a fundo em pesquisas acadêmicas (HENDERSON, 1979; JACOBSON; AAKER, 1985; KOTLER; SINGH, 1981; KUMAR, 2004; SHETH; SISODIA, 1995a, 1995b), principalmente a partir da criação do Profit Impact of Market Strategy - PIMS, como pode ser observado a seguir, procurando ampliar o entendimento entre investimento e desempenho no mercado. Em comum esses estudos focaram uma tríade formada pelos construtos: investimento, marketing mix e market share (LUKAS; WHITWELL; DOYLE, 2005; RUST et al., 2004; RUST; LEMON; ZEITHAML, 2004; SZYMANSKI; BHARADWAJ; VARADARAJAN, 1993).

O projeto Profit Impact of Market Strategy - PIMS foi uma iniciativa da General Eletric - GE e da Harvard Management Institute no início dos anos 1970 e depois pelo The Strategic Planning Institute, após 1975 (STRATEGIC PLANNING INSTITUTE, 2009). Tratava-se de uma pesquisa de grande proporções que envolveu o levantamento, entre 1970 e 1983, de informações de cerca de 3.000 (três mil) Stragic Business Units - SBU nos EUA. Cada SBU forneceu informações de seus mercados incluindo os produtos e a eficácia da estratégia implementada (BUZZELL; GALE, 1987; BUZZELL; GALE; SULTAN, 1975; ROBINSON; FOMEL, 1986). As descobertas mostraram a necessidade de se concentrar, principalmente na tríade proposta no início desta seção: investimento, market share, marketing mix, acrescentando as questões de intensidade de cada um.

O modelo ícone de análise de mercado é, sem dúvida, a já mencionada Matriz BCG, criada para se estudar a relação entre market share e impacto no caixa da empresa com o objetivo de se tomar decisões de portfólio (HENDERSON, 1979). O apelo intuitivo da Matriz a tornou popular entre os gerentes e presença garantida nos planos estratégicos e currículos universitários. Entretanto, o instrumento não é capaz de resolver o problema de análise isolada de cada elemento do marketing mix e, portanto, esforços para entender esta questão continuavam sendo feitos.

Outro modelo popularizado por Ries e Trout (1986) e baseado em Kotler e Singh (1981) é o marketing warfare, ou a aplicação de princípios de estratégia militar para fins de disputas de mercado. A grande virtude desta abordagem foi introduzir para a comunidade de gerentes de marketing a possibilidade de que as disputas de mercado guardavam relação com princípios estratégicos generalizáveis. Por outro lado, tratava-se mais de um conjunto flexível 
de princípios que, embora considerasse a tríade já mencionada não resolvia a questão do marketing metrics.

Procurando direcionar os pesquisadores de Marketing para projetos que buscassem cobrir pelo menos em parte esta lacuna, o Marketing Science Institute (1998) estabeleceu uma agenda de pesquisas que tinha o Marketing Metrics - como foi denominado o conjunto de iniciativas para mensurar o desempenho das empresas em relação às atividades de Marketing como prioridade para o triênio de 1998-2000.

No Brasil são poucos os estudos deste campo. Grinberg e Luce (2000, p. 7) fizeram um levantamento do estado-da-arte das principais linhas envolvidas na Europa e Estados Unidos e mostraram que os "alicerces da questão eram as medidas de performance e o grau de orientação para o mercado”. Desde esta iniciativa o campo permaneceu pouco explorado, e somente muitos anos depois Ribeiro et. al. (2006, p. 7) postularam que "percebe-se na literatura ainda oportunidade para estudos que visem revisar as escalas para mensurar competências, explorar as relações com performance”.

Procurando reduzir esta lacuna e contribuir para o debate neste campo, foi desenvolvida uma pesquisa que buscou evidências de relações entre investimento no marketing mix e market share de empresas do segmento químico-farmacêutico. Esta iniciativa tenta preencher uma lacuna dos estudos de marketing metrics no país. A pergunta que orientou a pesquisa foi: Qual a relação entre o grau de investimento no marketing mix das empresas e seu market share? Com isso pretende-se ter uma evidência de ambição estratégica de marketing.

Acredita-se ser este um primeiro passo para outras pesquisas no campo das métricas de Marketing que permitam estabelecer correlações entre programas de marketing e desempenho empresarial. O trabalho está organizado da seguinte forma: segue-se uma revisão da literatura pertinente ao tema, em seguida a metodologia aplicada a este trabalho, os principais resultados do estudo, a discussão dos resultados e as considerações finais.

\section{REVISÃO DA LITERATURA}

Os profissionais de Marketing, hoje, mais do que nunca estão sob intensa pressão para demonstrar que suas atividades contribuem positivamente para o desempenho das empresas (O’SULLIVAN; ABELA, 2007). Esta questão se agravou pela ausência de um corpo de métricas consolidadas que permitam entender a contribuição de cada ação desencadeada no mercado em seu esforço competitivo (KUMAR, 2004). A pressão por melhores práticas de mensuração ocorre não só na área dos negócios (AMBLER; KOKKINAKI; PUNTONI, 2004; FARRIS et. al., 2007) como também na acadêmica (LUKAS; WHITWEEL; DOYLE, 2005; RUST et al., 2004; RUST; LEMON; ZEITHAML, 2004).

Embora a proposta de uma agenda para estas pesquisas tenha sido estabelecida pelo Marketing Science Institute para o triênio de 1998-2000, muito pouco se avançou neste campo nos últimos anos (O'SULLIVAN; ABELA, 2007). As pesquisas diretamente ligadas às ações do mix de marketing das empresas foram pioneiras em estabelecer alguns critérios que seriam 
utilizados posteriormente e muitas questionaram a eficácia das medidas baseadas na relação marketing mix - desempenho (JACOBSON, 1988, 1990; JACOBSON; AAKER, 1985).

Os diferentes resultados destas pesquisas mostraram a necessidade de se estabelecer melhores critérios e modelos de análise que permitissem comparação de resultados (SZYMANSKI; BHARADWAJ; VARADARAJAN, 1993) o que acabou estimulando novos estudos que fossem além das limitações isoladas do marketing mix. Para isso foi necessário dar um passo atrás nas tradicionais variáveis funcionais acrescentando variáveis estratégicas no campo de pesquisa.

A clássica literatura de Marketing há muito prega que entregar um valor distinto e maior, do ponto de vista do cliente, é fundamental para adquirir vantagem competitiva sobre os concorrentes que não o fazem. Particularmente Day e Wensley (1988) estabeleceram a noção de vantagem competitiva e sua relação com medidas de desempenho que ultrapassem o market share como satisfação e lealdade dos clientes e a lucratividade. Outros autores desenvolveram melhor este postulado (DESHPANDÉ; FARLEY; WEBSTER JUNIOR, 1993; JAWORSKI; KHOLI, 1993; KOKKINAKI; AMBLER, 1999; NARVER; SLATER, 1990; SLATER; NARVER, 1995) estabelecendo um marco para os estudos na linha da orientação para o mercado - OPM.

Especificamente este postulado pode ser definido como o propósito de criar um valor superior para o comprador apoiado em procedimentos típicos para o mercado como orientação para os clientes e competidores, assim como aqueles ligados à organização interna, como a coordenação interfuncional (KOHLI; JAWORSKI, 1990; NARVER; SLATER, 1990). Para Slater e Narver (1995) tais funções estão positivamente relacionadas ao crescimento das vendas e com o sucesso de novos lançamentos no mercado. Portanto, a geração e a disseminação de informações e o estabelecimento de métricas assumem posição de destaque no desempenho das organizações, uma vez que propiciam as bases para o direcionamento das ações (KOHLI; JAWORSKI, 1990; MULLER, 2005).

Pesquisadores brasileiros como Tavares, Pinto e Afonso (2006) reconheceram que a OPM deve ser antecedida por informações que permitam reconhecer a multiplicidade de produtos e serviços que as pessoas consomem para cumprir determinadas tarefas, atendendo a diferentes objetivos, em uma seqüência de atividades inseridas no sistema de consumo, reduzirão as chances de fracasso. Segundo os autores é possível identificar três níveis de informação: 1) ir além do comportamento de compra buscando aprofundar o comportamento de uso, 2) procurar entender o sistema total de consumo, por meio do share-of-wallet (portfólio de compras do cliente) e, 3) analisar o sistema de consumo em novos detalhes derivados de outros subsistemas, coloca em relevo diferentes tipos de pessoas fazendo diferentes usos do mesmo produto ou diferentes produtos usados em situações similares (MOYSÉS FILHO et. al, 2008).

Para alguns autores com visão de coordenação inter funcional, geralmente, analisamse indicadores como geração de caixa, lucro e rentabilidade, volume de vendas e participação de mercado (BROWN; EISENHARDT, 1995; SONG; PARRY, 1997; URDAN; OSAKU, 2005). Estudos empíricos buscaram confirmar as diversas combinações de variáveis do OPM 
para alcançar o melhor desempenho. As pesquisas de Vorhies e Harker (1999, 2000) com 87 empresas mostraram que as empresas orientadas para mercado operam em alto nível com 6 tipos de competências aplicadas em seu marketing mix: desenvolvimento de produtos, planejamento, gestão e pesquisas de marketing, preço, canal de distribuição e promoção. Esta fórmula levava a um desempenho melhor que os concorrentes. Em uma recente pesquisa, Cooper, Edgett e Kleinschmidt (2004) verificaram que muitas das melhores práticas estão associadas à cultura e clima organizacionais voltados à inovação, à equipe envolvida diretamente com a inovação de produtos.

Uma atualização feita por Vorhies e Morgan (2005) propôs uma ampliação daquelas 6 competências para 8, incluindo: desenvolvimento de produtos, planejamento de marketing, gestão da informação de marketing, implementação de marketing, vendas, canal de distribuição, preço e comunicação de marketing.

No Brasil foram testados alguns dos modelos propostos pela literatura estrangeira por Urdan e Osaku (2005) a partir de 4 construtos principais: a) vantagem relativa do produto (COOPER, 1996); b) apreçamento orientado frente à concorrência; c) entrega de valor (DOLAN; SIMON, 1996); e, d) orientação ao consumidor, que parece ser um mote bem assimilado, embora não sendo uma unanimidade.

Uma pesquisa desenvolvida por Wheelwright e Clark (1994) para novos produtos mostrou que as empresas mais eficientes apresentavam seqüência de atividades individuais, estrutura e gerenciamento de projeto. O ponto de vista dos autores é de que: objetivos claramente definidos, foco no mercado, integração entre ambiente interno e externo, protótipos de alta qualidade e uma forte liderança refletem na capacidade das empresas trabalharem eficientemente o desenvolvimento de novos produtos atrativos bem como nos novos processos de produção dos mesmos. A vantagem competitiva resulta principalmente da execução (MOYSÉS FILHO et. al., 2008).

No Brasil, Perin e Sampaio (2003) demonstraram que a OPM é um pré requisito fundamental para as empresas quando aliada ao seu porte. Em estudo longitudinal aplicado ao setor Eletro Eletrônico do Brasil, os autores puderam constatar uma assimetria nos resultados obtidos por meio de porte da empresa e orientação para o mercado. O porte empresarial surte melhor efeito quando analisado em um determinado período enquanto o segundo caso resulta em melhores resultados ao longo de um período (p.11). Assim, eles concluem que "a orientação para o mercado exerce influência mais expressiva e homogênea sobre a performance empresarial” (PERIN; SAMPAIO, 2003, p. 11).

Para o mercado é crucial utilizar os dados resultantes das atividades competitivas como componente decisório. As informações geradas pelas ações de venda e as reações de concorrentes e compradores permitem estabelecer correlações e a elaboração de mapas de decisão mais seguros. Do lado da academia foi necessário esperar a década de 1990 para que alguns estudos, como os de Sheth e Sisodia (1995 a; 1995 b) mostrassem a relação baixa e até mesmo negativa entre gastos de Marketing e desempenho financeiro com base somente na relação market share - performance (FARRIS et. al., 2007). 


\subsection{O conceito de ambição estratégica de marketing}

Dentro deste mesmo debate, que já dura desde os anos 1970, Cook Junior (1983, 1985) buscou verificar a relação entre o desempenho do marketing mix e seu impacto no market share, introduzindo o conceito de ambição estratégica de marketing. Para ele em um mercado competitivo o comportamento do concorrente pode ser medido pela intensidade com ele aplica seus investimentos no mercado. Este indicador pode informar se sua estratégia é defensiva ou expansionista. Assim, para um dado mercado, o total de investimento de marketing (E) de todos os concorrentes é a soma do investimento isolado de cada empresa (e) adicionado ao investimento dos concorrentes. Isto pode ser considerado uma primeira relação e é expressa pela fórmula: $E=\mathrm{e}+\mathrm{e}^{*}$.

Se uma determinada empresa, por exemplo, quintuplica seus investimentos de marketing pode ser fácil argumentar que se trata de uma estratégia agressiva. Mas somente este dado isolado é insuficiente, sendo necessário saber se este não é um movimento geral dos concorrentes. Este ponto leva o autor a concluir que a estratégia implica em uma relação formal entre a ambição dos competidores e o investimento isolado de cada um. Assim, o investimento estratégico (e) é o produto aritmético de um investimento isolado e os investimentos dos competidores, o que é expresso na fórmula: e = ye*, na qual o valor de y é a ambição estratégica, ou no exemplo acima, a empresa tem um valor de y equivalente a 0,05. Caso ela estivesse na média seu investimento seria de 0,01. Trata-se de uma medida simples que pode fornecer uma métrica de mercado e aponta para a evidência de que a clássica premissa da relação de desempenho entre marketing mix e market share necessita ser mediada pela decisão estratégica e os efeitos de longo prazo (p.73).

Como mencionado antes Cook Junior $(1983,1985)$ procurou distinguir as tradicionais relações do desempenho com o market share trabalhando o conceito de ambição estratégica e sua relação com a vontade de investir. O market share deveria ser analisado a partir da ponderação com as decisões estratégicas e de investimento. Seu potencial de informar a posição de uma empresa não deveria ser encarado isoladamente e tampouco com uma visão de curto prazo como medida do sucesso nos negócios. Sua base de análise foi o mercado automobilístico norte-americano entre 1975-1980; segundo o autor, os produtos nacionais perderam para os importados devido à adoção de uma política de colheita dos lucros por parte dos produtos norte-americanos e, da ambição dos importados em bancar investimentos em cada elemento do marketing mix (mais investimento em produto, distribuição e promoção e preço baixo), para ganhar market share no longo prazo.

Pelo exposto na literatura pesquisada percebe-se que as dimensões podem ser visualizadas em ambos os níveis: estratégico e organizacional. O nível estratégico compreende o alinhamento de objetivos e metas da empresa, considerando as particularidades das empresas, com o desenvolvimento conjunto de projetos a serem desenvolvidos. O nível organizacional é relativo ao desenvolvimento específico das ações, ou seja, é responsável pelos aspectos gerenciais. Com esta fundamentação teórica foi desenvolvida a pesquisa cuja metodologia é descrita a seguir. 


\section{MÉTODO}

\subsection{Escalas}

Este estudo, de natureza quantitativa, transversal e exploratória, utilizou para mensuração das variáveis do marketing mix a escala proposta por Cook Junior (1983), que as analisa em quatro dimensões distintas. A primeira dimensão é composta por oito variáveis para mensuração do investimento feito pela empresa no tocante a concepção e desenvolvimento de produtos, a segunda é composta por sete variáveis para mensuração do grau de investimento realizado em promoção de vendas. O terceiro bloco de variáveis, no total de oito, identifica o esforço da empresa nas atividades de distribuição de seus produtos e, finalmente, o quarto bloco, constituído por sete variáveis, mensura o foco destinado à política de comercialização da empresa (apêndice A).

Pela proposta de Cook Junior (1983), os respondentes posicionam o grau de investimento realizado por sua organização, em cada uma das trinta variáveis, comparando seu esforço com o realizado pela concorrência, por meio de uma escala de dez pontos. O respondente deve assumir que o investimento realizado pela concorrência no marketing mix tem base 100 e posicionar o investimento que sua organização realiza entre $0 \%$ e $300 \%$ (ou mais).

\subsection{Questionário}

Os autores optaram pela utilização de um formulário de pesquisa eletrônico estruturado em três seções distintas. A primeira seção continha questões que possibilitavam classificar o segmento econômico da empresa respondente, o nível que o entrevistado possuía dentro da hierarquia da organização, bem como sua área de atuação. A segunda seção, dividida em quatro blocos apresentava aos respondentes a escala de mensuração do marketing mix de Cook Junior (1983) e, finalizando o questionário, a terceira seção solicitava que o respondente informasse o market share que a empresa possuía. Este último dado serviu para testar a validade nomológica da escala, ou seja, até que ponto construtos teoricamente distintos se relacionam de maneira significativa empiricamente (LEVIN; FOX, 2006).

\subsection{Amostra}

As empresas convidadas a participar deste estudo fazem parte do rol de fornecedores de uma empresa multinacional do ramo químico-farmacêutico que figura entre as vinte maiores companhias do setor. O questionário foi enviado eletronicamente para seiscentas empresas escolhidas por conveniência, o que resultou em uma amostra não-probabilistica.

\subsection{Procedimentos}

Visando possibilitar a participação de respondentes de empresas sediadas em outros países, o questionário foi disponibilizado nas versões em português e inglês. Elaborou-se convite aos participantes, explicando o intuito e a motivação acadêmica do estudo. Para potencializar o desejo de participação, os convites foram enviados, em formato eletrônico nominal aos participantes, que tinham a opção de escolher a versão de questionário preferida. A pesquisa empírica foi realizada entre janeiro e março de 2009. 


\subsection{Plano de Análise dos dados}

Para a análise dos dados realizou-se a análise fatorial exploratória (AFE) e regressão linear multivariada com a utilização do SPSS for Windows na versão 13.0.

A AFE deu-se pelo método de análise de componentes principais e rotação ortogonal Varimax. Mesmo que as dimensões da escala de Cook Junior (1983) pressuponham intercorrelações positivas, o que permitiria a utilização de rotação oblíqua, os autores optaram pela rotação ortogonal por esta metodologia testar com maior rigor a unidimensionalidade das variáveis. Nesta fase também foi feita a análise de validade interna por meio do cálculo do coeficiente Alfa de Cronbach.

Os seguintes critérios foram adotados para a AFE: comunalidade extraída dos itens superior a 0,4, teste de esfericidade de Bartlett significativo ao nível de 5\% e teste KaiserMeyer-Olkin (KMO) superior a 0,7 (HAIR JUNIOR et. al., 2005). Para o coeficiente de Alfa de Cronbach o valor mínimo aceitável foi 0,7 (NUNNALLY, 1978).

O modelo regressão linear multivariada deu-se pelo método Enter, tendo como variável dependente o market share informado e, como variáveis independentes, o marketing mix sob a ótica da escala de Cook Jr.(1983). O modelo de regressão linear buscou identificar o coeficiente de determinação $\left(r^{2}\right)$ que é a relação entre a variação explicada e a variação total entre as variáveis independentes e a dependente.

\section{RESULTADOS DA PESQUISA}

Para atender aos pressupostos da AFE e da regressão linear multivariada, realizou-se uma análise preliminar dos dados. O percentual máximo de missing values (dados ausentes) por item foi de 2,14\%, e o percentual médio de 1,01\%. Diante disso, adotou-se o procedimento de substituição dos missing values pela média.

A verificação da homocedasticidade e da linearidade da amostra foi feita por meio das distribuições residuais e da análise dos gráficos de dispersão bivariados (HAIR JUNIOR et. al., 2005), verificando-se a sua existência dentro dos limites adequados para a realização da AFE.

Por meio da análise da matriz antiimagem, observou-se medidas de adequação amostral (MSA) que variaram entre 0,78 e 0,91 indicando a fatorabilidade da amostra.

Por fim, a existência de outliers foi verificada por meio da análise do gráfico Boxplot e indicou a necessidade de eliminação de 8 observações da amostra, como será visto à seguir.

\subsection{Perfil da amostra}

Das 600 empresas convidadas a participar da pesquisa 122 retornaram os questionários (20,33\%), todavia apenas 114 foram consideradas válidas após a exclusão de 8 outliers, como demonstrado na Figura 1. 


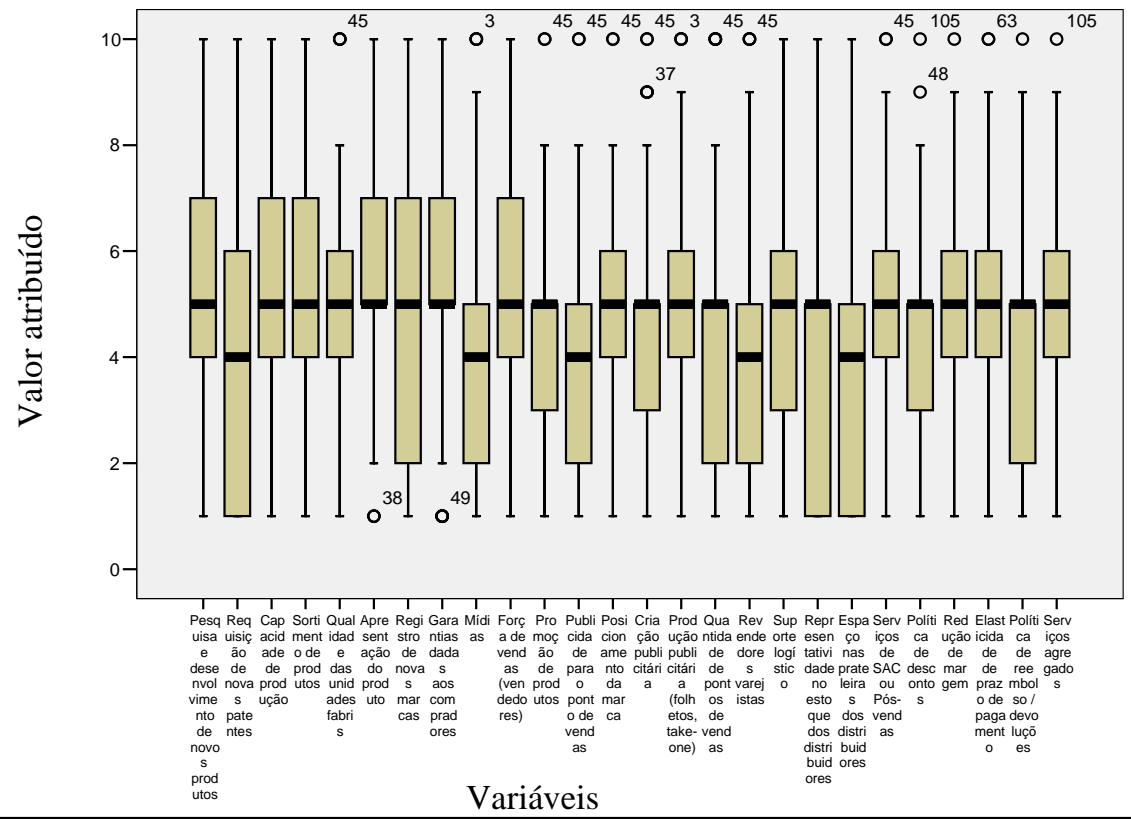

Figura 1 - Outliers da amostra

Fonte: Elaborado pelos autores

Da amostra válida de 114 empresas, 77 (67,5\%) são brasileiras, 28 (24,6) são sediadas nos Estados Unidos e 9 sediadas em outros países. Em relação ao segmento econômico de atuação das organizações participantes, 65 (57\%) são empresas comerciais ou de serviços, 45 $(39,5 \%)$ industriais e somente 4 (3,5\%) pertencem ao segmento de agrobusiness.

Quanto ao perfil dos respondentes 53 (46,5\%) possuem nível gerencial e, 32 (28,1\%) nível de direção, sendo que 42 respondentes (36,8\%) militam na área comercial e 37 (32,5\%) nas áreas de suporte (recursos humanos, contabilidade, departamento fiscal, dentre outras). Essas distribuições estão detalhadas na Tabela 1.

Tabela 1 - Distribuição da amostra

\begin{tabular}{l|l|c|c}
\hline & & $\mathbf{n}$ & $\mathbf{\%}$ \\
\hline & Industrial & 45 & 39,47 \\
\hline Segmento & Comércio/Serviços & 65 & 57,02 \\
\hline & Agronegócios & 4 & 3,51 \\
\hline & Total & $\mathbf{1 1 4}$ & $\mathbf{1 0 0 , 0 0}$ \\
\hline & Operacional & 17 & 14,91 \\
\hline Nível & Supervisão & 12 & 10,53 \\
\hline & Gerencial & 53 & 46,49 \\
\hline & Direção & 32 & 28,07 \\
\hline & Total & $\mathbf{1 1 4}$ & $\mathbf{1 0 0 , 0 0}$ \\
\hline & Produção & 18 & 15,79 \\
\hline & Comercial & 42 & 36,84 \\
\hline Área & Distribuição & 17 & 14,91 \\
\hline & \multicolumn{2}{|c}{}
\end{tabular}




\begin{tabular}{c|l|c|c}
\hline & Suporte & 37 & 32,46 \\
\hline & Total & $\mathbf{1 1 4}$ & $\mathbf{1 0 0 , 0 0}$ \\
\hline
\end{tabular}

Fonte: Elaborado pelos autores

O tempo médio utilizado para preenchimento do questionário eletrônico foi de 8,7 minutos ( $\sigma=13$ minutos).

\subsection{Análise fatorial exploratória}

A AFE sugeriu a eliminação de seis variáveis da escala, sendo quatro por baixa comunalidade $(<0,4)$ e duas por carga fatorial cruzada entre dois fatores. Após a eliminação das seis variáveis do modelo original, realizou-se nova AFE com as vinte e quatro variáveis restantes. Nesta nova configuração, o teste de Bartlett foi significativo a $1 \%\left(\chi^{2}=1930,19\right.$; $276 d f ; \mathrm{p}<0,01)$ e o $\mathrm{KMO}=0,889$ o que indica excelente adequabilidade para análise de componentes principais (HAIR et. al., 2005). A Tabela 2 demonstra o resultado da análise fatorial.

Tabela 2 - Resultado da AFE

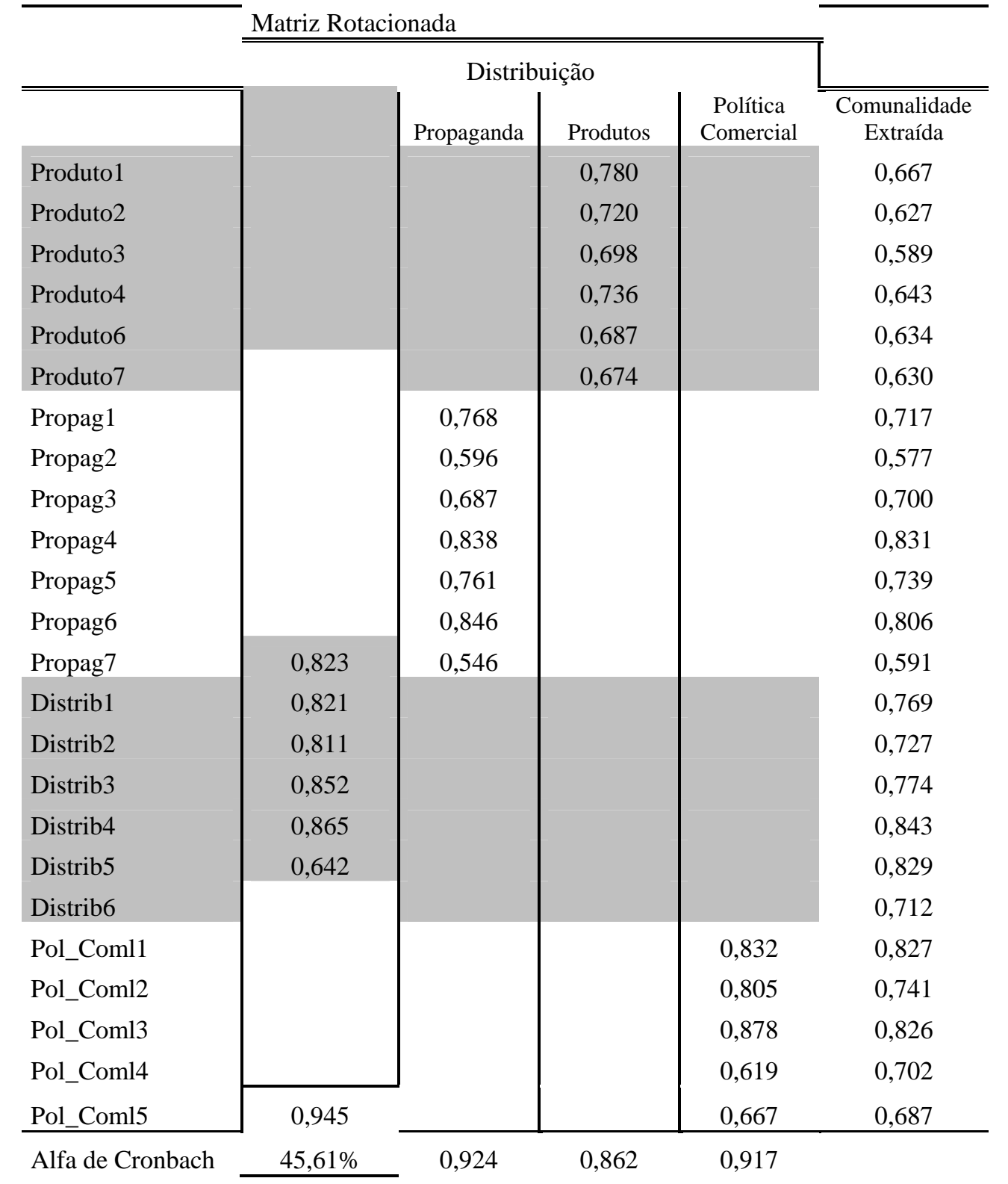


Variância explicada

Método de extração: Análise de componentes principais

Método de rotação: Varimax com normalização Kaiser

Fonte: Elaborado pelos autores

Como era esperado, foram identificados quatro fatores com eigenvalues superiores a 1 , cargas fatoriais superiores a 0,546 e variância total explicada de 71,19\%. As comunalidades extraídas ficaram entre 0,577 e 0,843 e a validade interna ficou evidenciada por coeficientes Alfa de Cronbach entre 0,862 e 0,945.

Como demonstrado na Tabela 2, o agrupamento fatorial das variáveis seguiu a modelagem teórica elaborada por Cook Jr. (1983) formando quatro fatores: 1) um composto pelas variáveis de produtos; 2) outro composto com as variáveis de distribuição; 3) um terceiro, com as variáveis de propaganda e; 4) o restante agrupando as variáveis da política comercial.

A Tabela 3 apresenta a frequência relativa das respostas obtidas na pesquisa de campo. Tabela 3 - Distribuição das respostas

\begin{tabular}{|c|c|c|c|c|c|c|c|c|c|c|c|}
\hline \multicolumn{12}{|c|}{ Percentis das respostas } \\
\hline Escala & 1 & 2 & 3 & 4 & 5 & 6 & 7 & 8 & 9 & 10 & Médias e desvios \\
\hline Variáveis & $\mathbf{0}$ & 25 & 50 & 75 & 100 & 125 & 150 & 200 & 250 & 300 ou + & padrão \\
\hline Produto1 & 4,50 & 9,01 & 9,91 & 13,51 & 20,72 & 9,91 & 13,51 & 9,91 & 3,60 & 5,41 & $5,30 \pm 2,34$ \\
\hline Produto2 & 30,56 & 11,11 & 4,63 & 8,33 & 18,52 & 4,63 & 8,33 & 8,33 & 1,85 & 3,70 & $3,99 \pm 2,75$ \\
\hline Produto3 & 7,21 & 5,41 & 9,91 & 18,02 & 20,72 & 9,01 & 11,71 & 9,91 & 3,60 & 4,50 & $5,16 \pm 2,32$ \\
\hline Produto4 & 10,19 & 7,41 & 7,41 & 10,19 & 22,22 & 13,89 & 13,89 & 4,63 & 5,56 & 4,63 & $5,13 \pm 2,43$ \\
\hline Produto6 & 4,63 & 6,48 & 3,70 & 12,04 & 27,78 & 15,74 & 12,96 & 9,26 & 3,70 & 3,70 & $5,45 \pm 2,11$ \\
\hline Produto7 & 22,43 & 4,67 & 4,67 & 13,08 & 17,76 & 9,35 & 12,15 & 11,21 & 2,80 & 1,87 & $4,62 \pm 2,60$ \\
\hline Propag1 & 13,51 & 14,41 & 19,82 & 13,51 & 18,02 & 7,21 & 8,11 & 0,00 & 2,70 & 2,70 & $3,97 \pm 2,21$ \\
\hline Propag2 & 3,60 & 9,91 & 9,91 & 12,61 & 23,42 & 12,61 & 14,41 & 8,11 & 2,70 & 2,70 & $5,14 \pm 2,13$ \\
\hline Propag3 & 12,84 & 11,01 & 17,43 & 11,93 & 24,77 & 10,09 & 4,59 & 4,59 & 0,00 & 2,75 & $4,16 \pm 2,14$ \\
\hline Propag4 & 14,68 & 13,76 & 12,84 & 18,35 & 26,61 & 3,67 & 5,50 & 1,83 & 0,00 & 2,75 & $3,90 \pm 2,05$ \\
\hline Propag5 & 7,41 & 9,26 & 9,26 & 12,04 & 26,85 & 13,89 & 12,04 & 5,56 & 0,00 & 3,70 & $4,85 \pm 2,14$ \\
\hline Propag6 & 10,09 & 11,01 & 11,01 & 16,51 & 25,69 & 11,93 & 5,50 & 0,92 & 4,59 & 2,75 & $4,46 \pm 2,19$ \\
\hline Propag7 & 9,01 & 7,21 & 9,91 & 12,61 & 31,53 & 12,61 & 7,21 & 4,50 & 2,70 & 2,70 & $4,75 \pm 2,13$ \\
\hline Distrib1 & 21,62 & 6,31 & 10,81 & 9,91 & 28,83 & 8,11 & 6,31 & 2,70 & 0,00 & 5,41 & $4,19 \pm 2,43$ \\
\hline Distrib2 & 25,24 & 4,85 & 10,68 & 11,65 & 28,16 & 4,85 & 7,77 & 1,94 & 0,97 & 3,88 & $4,01 \pm 2,40$ \\
\hline Distrib3 & 20,72 & 3,60 & 5,41 & 11,71 & 26,13 & 11,71 & 9,01 & 6,31 & 2,70 & 2,70 & $4,57 \pm 2,45$ \\
\hline Distrib4 & 28,70 & 4,63 & 6,48 & 9,26 & 28,70 & 7,41 & 6,48 & 3,70 & 0,93 & 3,70 & $4,03 \pm 2,49$ \\
\hline Distrib5 & 32,08 & 5,66 & 5,66 & 6,60 & 33,02 & 5,66 & 3,77 & 3,77 & 1,89 & 1,89 & $3,78 \pm 2,41$ \\
\hline Distrib6 & 11,93 & 3,67 & 5,50 & 8,26 & 35,78 & 12,84 & 7,34 & 8,26 & 3,67 & 2,75 & $5,03 \pm 2,24$ \\
\hline Pol_Coml1 & 12,61 & 9,91 & 9,91 & 13,51 & 31,53 & 9,91 & 5,41 & 3,60 & 2,70 & 0,90 & $4,33 \pm 2,08$ \\
\hline Pol_Coml2 & 9,91 & 9,01 & 9,01 & 12,61 & 29,73 & 11,71 & 9,01 & 4,50 & 3,60 & 0,90 & $4,65 \pm 2,12$ \\
\hline Pol_Coml3 & 8,26 & 6,42 & 5,50 & 12,84 & 38,53 & 11,93 & 6,42 & 3,67 & 4,59 & 1,83 & $4,87 \pm 2,04$ \\
\hline Pol_Coml4 & 16,51 & 11,93 & 2,75 & 5,50 & 42,20 & 7,34 & 5,50 & 3,67 & 3,67 & 0,92 & $4,36 \pm 2,23$ \\
\hline Pol_Coml5 & 5,56 & 6,48 & 10,19 & 7,41 & 38,89 & 7,41 & 10,19 & 7,41 & 4,63 & 1,85 & $5,08 \pm 2,08$ \\
\hline Média & 14,32 & 8,05 & 8,85 & 11,75 & 27,75 & 9,73 & 8,63 & 5,35 & 2,63 & 2,94 & $4,57 \pm 2,33$ \\
\hline D.Padrão & 8,55 & 3,11 & 4,07 & 3,23 & 6,67 & 3,26 & 3,23 & 3,07 & 1,63 & 1,29 & \\
\hline
\end{tabular}

Fonte: Elaborado pelos autores 
Em 42,9\% das avaliações realizadas foi sinalizado que a concorrência tem maior foco competitivo no marketing mix quando comparada com a empresa respondente, enquanto em outros $27,75 \%$ a avaliação apontou equilíbrio no esforço realizado pelos players.

Interessante notar que o grupo de menor média foi o de Distribuição $(\mu=4,26)$ e o de maior expressão foi o grupo das variáveis de Produto $(\mu=4,94)$. Do mesmo modo, as variáveis Distrib5 e Propag4 tiveram menores médias $(\mu=3,78 / \sigma=2,41$ e $\mu=3,90 / \sigma=2,05$ respectivamente) indicando que, na opinião dos respondentes, o espaço ocupado pelos produtos da empresa nos distribuidores/pontos de vendas e a publicidade nos pontos de vendas não são atributos de destaque nas organizações pesquisadas.

Diante destes resultados, uma matriz de correlação bivariada foi elaborada com o intuito de verificar a relação entre a área de atuação dos respondentes e sua avaliação das variáveis do marketing mix. A Figura 2 demonstra que não há correlação significativa entre estas variáveis indicando que as avaliações não foram tendenciosas.

\section{Correlações}

\begin{tabular}{|c|c|c|c|c|c|c|}
\hline & & $\begin{array}{l}\text { Área de } \\
\text { atuação do } \\
\text { respondente }\end{array}$ & Média produto & $\begin{array}{c}\text { Média } \\
\text { propaganda }\end{array}$ & $\begin{array}{c}\text { Média } \\
\text { distribuição }\end{array}$ & $\begin{array}{l}\text { Média política } \\
\text { comercial }\end{array}$ \\
\hline \multirow{3}{*}{$\begin{array}{l}\text { Qual sua área } \\
\text { de atuação? }\end{array}$} & r de Pearson & 1 & ,016 & ,041 &,- 033 & ,125 \\
\hline & significância & & ,866 & ,664 & ,732 & 187 \\
\hline & $\mathrm{N}$ & 114 & 114 & 113 & 113 & 113 \\
\hline \multirow[t]{3}{*}{ Média produto } & r de Pearson & ,016 & 1 & ,638** &, $537 * \star$ &, $517^{\star \star}$ \\
\hline & significância & ,866 & & ,000 &, 000 & ,000 \\
\hline & $\mathrm{N}$ & 114 & 114 & 113 & 113 & 113 \\
\hline \multirow{3}{*}{$\begin{array}{l}\text { Média } \\
\text { propaganda }\end{array}$} & r de Pearson & ,041 & ,638** & 1 & ,595** &, $626^{\star \star}$ \\
\hline & significância & ,664 & ,000 & &, 000 & ,000 \\
\hline & $\mathrm{N}$ & 113 & 113 & 113 & 112 & 112 \\
\hline \multirow{3}{*}{$\begin{array}{l}\text { Média } \\
\text { distribuição }\end{array}$} & r de Pearson &,- 033 &, $537^{\star \star}$ &, $595^{\star \star}$ & 1 &, $582^{\star \star}$ \\
\hline & significância & ,732 & ,000 & ,000 & & ,000 \\
\hline & $\mathrm{N}$ & 113 & 113 & 112 & 113 & 113 \\
\hline \multirow{3}{*}{$\begin{array}{l}\text { Média política } \\
\text { comercial }\end{array}$} & r de Pearson & ,125 &, $517^{\star \star}$ & ,626** &, $582^{\star \star *}$ & 1 \\
\hline & significância & 187 & ,000 & ,000 &, 000 & \\
\hline & $\mathrm{N}$ & 113 & 113 & 112 & 113 & 113 \\
\hline
\end{tabular}

**. Correlações significativas ao nível de $1 \%$ (teste bicaudal)

Figura 2 - Matriz de correlação bivariada entre área e média das avaliações

Fonte: Elaborado pelos autores

\subsection{Modelo de regressão linear multivariada}

Utilizando os grupos formados na AFE como variáveis independentes e o market share como variável dependente do modelo de regressão linear multivariada, encontrou-se o coeficiente de determinação $\mathrm{r}^{2}=0,206$, o que indica que as variáveis do marketing mix, sob a ótica de Cook Jr. (1983), explicam 20,6\% da variação do market share das empresas participantes do presente estudo $(F(4)=3,851 ; p<0,01)$, como demonstrado nas Figuras 3 e 4.

\begin{tabular}{|c|c|c|c|c|c|}
\hline & Soma dos Quadrados & $\begin{array}{c}\text { Graus de } \\
\text { liberdade }\end{array}$ & Quadrados médios & $\mathrm{F}$ & $\mathrm{p}$ \\
\hline Regressão & 0,218 & 4 & 0,054 & 3,851 & $\mathrm{p}<1 \%$ \\
\hline
\end{tabular}




\begin{tabular}{|c|c|c|c|c|c|}
\hline Resíduo & 4,735 & 74 & 0,064 & & \\
\hline Total & 4,952 & 78 & & & \\
\hline
\end{tabular}

\begin{tabular}{|l|c|c|c|c|c|}
\cline { 2 - 6 } \multicolumn{1}{c|}{} & \multicolumn{2}{c|}{ Coeficientes não padronizados } & Coeficientes padronizados & $\mathrm{t}$ & $\mathrm{p}$ \\
\cline { 2 - 6 } \multicolumn{1}{c|}{} & Beta & Erro padronizado & Beta & & \\
\hline $\begin{array}{l}\text { Variáveis } \\
\text { independentes }\end{array}$ & 0,154 & 0,101 & & 4,532 & $\mathrm{p}<1 \%$ \\
\hline MMix_produto & 0,024 & 0,023 & 0,171 & 4,053 & $\mathrm{p}<1 \%$ \\
\hline MMix_propaganda & 0,017 & 0,024 & 0,122 & 2,071 & $\mathrm{p}<10 \%$ \\
\hline MMix_distribuição & 0,023 & 0,018 & 0,179 & 3,248 & $\mathrm{p}<5 \%$ \\
\hline MMix_polcoml & $-0,011$ & 0,021 & $-0,072$ & 3,508 & $\mathrm{p}<5 \%$ \\
\hline Variável dependente: Market Share informado
\end{tabular}

Figura 3- Coeficientes da regressão linear

Fonte: os autores

\begin{tabular}{|l|c|c|c|c|}
\hline Modelo & $\mathrm{r}$ & $\mathrm{r}^{2}$ & $\mathrm{r}^{2}$ ajustado & $\begin{array}{c}\text { Erro de estimativa } \\
\text { padronizado }\end{array}$ \\
\hline 1 &, 454 &, 206 &, 199 &, 25294 \\
\hline
\end{tabular}

Figura 4 - Coeficiente de determinação da regressão

Fonte: Elaborado pelos autores

\section{DISCUSSÃO DOS RESULTADOS}

A escala proposta por Cook Junior (1983) mostrou-se válida e confiável, mesmo não sendo possível utilizar seis de suas variáveis originais. A análise fatorial exploratória comprovou a existência dos quatro fatores sugeridos por este autor - distribuição, propaganda, produtos e política comercial - demonstrando alta congruência entre os diversos itens que compõem cada uma das escalas.

Os respondentes apontaram para um intrigante posicionamento competitivo entre suas empresas e seus principais concorrentes, pois somente em $29 \%$ das avaliações, a empresa do respondente foi considera como a que realiza o maior investimento nas quatro dimensões do marketing mix estudado o que seria considerado, na taxionomia de Cook Junior (1983), como uma ambição estratégica de partida. Este baixo desempenho competitivo não está alinhado com o market share $(\mu=24,5 \%)$ da amostra estudada.

As análises dos respondentes podem estar impregnadas pelo desejo de criar valor superior aos clientes (KOHLI; JAWORSKI, 1990), pelo entendimento de que a empresa deveria ter maior orientação para o mercado e para os concorrentes, como teorizado por Slater e Narver (1995) ou ainda estarem relacionadas com o ditado americano que diz: the grass is always greener on the other side of the fence. De qualquer forma, é importante que os executivos fiquem atentos aos movimentos de seus concorrentes e que, como observado por Day (1994), utilizem estas informações para criação de modelos mentais de gestão vanguardistas. 
Uma questão a ser discutida é o entendimento do baixo investimento em divulgação dos produtos nos pontos de vendas, quer sejam estes somente distribuidores, ou varejistas. Como não foi identificada correlação significativa (1 e 5\%) entre a área de atuação do respondente e seu posicionamento - que demonstra isenção e tendência à generalização -, entende-se que esta lacuna é uma oportunidade de diferenciação entre os players e que poderá tornar-se um atributo ganhador de pedidos. Do mesmo modo, o aperfeiçoamento do aparato logístico da organização tornar-se-á, muito provavelmente, fonte de diferenciação frente aos demais competidores.

O modelo de regressão linear multivariada identificou que as dimensões do marketing mix, definidas pelas variáveis determinadas por Cook Junior (1983), explicaram 20,6\% da variação do market share das empresas pesquisadas. Embora significativo, o poder preditivo do modelo pode ser classificado como tímido (HAIR JUNIOR et. al., 2005). Entende-se então, que o coeficiente de determinação evidenciado demonstra que existe a possibilidade de explicação relacionada a outras variáveis não incluídas no modelo. Algumas indicações já constam na própria literatura revisada, como nos estudos de Narver e Slater (1990) e Jaworski e Kohli (1993).

Os autores também identificaram que o market share pode não ser o melhor estimador do desempenho organizacional e que outros indicadores, tais como retorno sobre investimentos, lucros sobre vendas e satisfação dos consumidores poderiam ser utilizados na validação nomológica das dimensões do marketing mix.

\section{CONSIDERAÇÕES FINAIS}

\subsection{Implicações acadêmicas}

Este estudo procurou reduzir uma lacuna no campo de métricas de Marketing no Brasil desenvolvendo uma pesquisa com o objetivo de entender como empresas da cadeia produtiva do segmento químico-farmacêutico trabalham a relação entre as escalas propostas por Cook Junior (1983), a saber: ambição estratégica de marketing, o esforço combinado dos elementos do marketing mix e seu efeito no market share. Sua aplicação se tornou a primeira contribuição acadêmica deste trabalho.

Outro ponto que deve ser considerado é confirmação empírica do pressuposto teórico da correlação positiva entre investimento no marketing mix e o market share da organização. Do mesmo modo, diante dos parcos estudos realizados sobre a relação entre investimento em marketing e desempenho empresarial, esse estudo vislumbrou jogar luz sobre um tema de relevância para a ciência administrativa em especial à administração estratégica de marketing, avançando na discussão dos conceitos de marketing metrics.

\subsection{Implicações gerenciais}

Do ponto de vista gerencial, esta pesquisa buscou identificar o foco e difusão dos investimentos realizados, por organizações de um setor específico, nos atributos do marketing 
mix. O estudo sugere que existem atributos, com grande potencial de diferenciação, ainda não totalmente explorados. Neste sentido, parece razoável sugerir que os gestores analisem os pontos abordados com o propósito de formularem novas estratégias visando corrigir os hiatos identificados, o que poderá ser traduzido em incremento do desempenho corporativo.

Do ponto de vista operacional, o estudo demonstra que a escala proposta por Cook Junior (1983) é parcimoniosa, objetiva e prática, podendo ser aplicada com a celeridade exigida pela dinâmica do cenário competitivo atual.

\subsection{Limitações e sugestões para novos estudos}

A característica da amostra já constitui uma das limitações deste trabalho. A natureza não probabilística impede a generalização dos resultados e o corte transversal utilizado na coleta de dados sugere que os resultados obtidos devam ser considerados sob a ótica das características específicas da amostra utilizada. Portanto, recomenda-se a replicação deste trabalho, utilizando-se o mesmo instrumento e um procedimento de coleta aleatório. Da mesma forma, uma aplicação em corte longitudinal conferirá maior robustez às análises.

Outra limitação a ser apontada é adoção de apenas um respondente por organização. Mesmo que as análises não evidenciaram parcialidade nas respostas, metodologicamente seria mais apropriado a obter a visão de mais de um integrante de cada empresa.

Por fim, espera-se que este estudo tenha contribuído, mesmo que de forma modesta, para o melhor entendimento da relação marketing mix - desempenho e que constitua inspiração para futuros trabalhos nesta área.

\section{REFERENCIAS}

AMBLER, T.; KOKKINAKI, F.; PUNTONI, S. Assessing marketing performance: reasons for metrics selection. Journal of Marketing Management, Fayetteville, v. 20, n. 3-4, p. 475-99, 2004.

BROWN, S.; EISENHARDT, K. Product development: past research, present findings, and future directions. The Academy of Management Review, Ada, v. 20, n. 2, p. 343-378, 1995.

BUZZELL, R. D.; GALE, B.T. The PIMS principles: linking strategy to performance. New York: Free Press, 1987.

BUZZELL, R.; GALE, B. T.; SULTAN, R. G. M. Market share - a key to profitability. Harvard Business Review, Boston, v. 53, n. 1, p. 97-106, 1975.

COOK JUNIOR, V. J. Marketing strategy and differential advantage. Journal of Marketing, New York, v. 47, n. 2, p. 68-75, 1983.

COOK JUNIOR, V. J. The net present value of market share. Journal of Marketing, New York, v. 49, n. 3, p. 49-63, 1985.

COOPER, R. Benchmarking firms new product performance and practice. Engineering 
Management Review, n. 23, p. 112-120, 1996.

COOPER, R. G.; EDGETT, S. J.; KLEINSCHMIDT, E. J. Benchmarking best NPD practices-1. Research Technology Management, Lancaster, v. 47, n. 1 p. 31-47, 2004.

DAY, G. S. The capabilities of market-driven organizations. Journal of Marketing, New York, v. 58, n. 4, p. 37-52, 1994.

DAY, G. S.; WENSLEY, R. Assessing advantage: a framework for diagnosing competitive superiority. Journal of Marketing, New York, v. 52, n. 2, p. 1-20, 1988.

DESHPANDÉ, R.; FARLEY, J. U.; WEBSTER JUNIOR, F. E. Corporate culture customer orientation, and innovativeness in Japanese firms: a quadrad analysis. Journal of Marketing, New York, v. 57, n. 1, p. 23-27, 1993.

DOLAN, R.; SIMON, H. Power pricing. New York: Free Press, 1996.

FARRIS, P. W. et al. Métricas de marketing: mais de 50 métricas que todo executivo deve dominar. Porto Alegre: Bookman, 2007.

GRINBERG, C. S.; LUCE, F. B. Marketing metrics: um novo desafio para a área de marketing. In: ENCONTRO DA ASSOCIAÇÃO NACIONAL DE PÓS-GRADUAÇÃO E PESQUISA EM ADMINISTRAÇÃO, 24., 2000, Florianópolis. Anais... Rio de Janeiro: ANPAD, 2000. 1 CD-ROM.

HAIR JUNIOR, J. F. et al. Análise multivariada de dados. Tradução Adonai Schlup Sant’Anna e Anselmo Chaves Neto. 5. ed. Porto Alegre: Bookman, 2005.

HENDERSON, B. D. Henderson on corporate strategy. Cambridge: Abt Books, 1979.

JACOBSON, R. Distinguishing among competing theories of the market share effect. Journal of Marketing, New York, v. 52, n. 4, p. 68-80, 1988.

JACOBSON, R. Unobservable effects and business performance. Marketing Science, Providence, v. 9, n. 1, p. 74-85, 1990.

JACOBSON, R.; AAKER, D. A. Is market share all that it's cracked up to be? Journal of Marketing, New York, v. 49, n. 4, p. 11-22, 1985.

JAWORSKI, B. J.; KOHLI, A. K. Market orientation: antecedents and consequences. Journal of Marketing, New York, v. 57, n. 3, p. 1-15, 1993.

KOHLI, A. K.; JAWORSKI, B. J. Market orientation: the construct, research propositions, and managerial implications. Journal of Marketing, New York, v. 54, n. 2, p. 1-18, 1990.

KOKKINAKI, F.; AMBLER, T. Marketing performance assessment: current practice and the role of firm orientation. London: London Business School, 1999. (Working Paper, n. 99- 
901).

KOTLER, P.; SINGH, R. Marketing warfare in the 1980's. Journal of Business Strategy, Boston, v. 1, n. 3, p. 30-41, 1981.

KUMAR, N. Marketing como estratégia: uma orientação inovadora e comprovada para o crescimento e a inovação. Rio de Janeiro: Elsevier, 2004.

LEVIN, J.; FOX, J. A. Estatística para ciências humanas. São Paulo: Person Prentice Hall, 2006.

LUKAS, B. A.; WHITWELL, G. J.; DOYLE, P. How can a shareholder value approach improve marketing's strategic influence? Journal of Business Research, New York, v. 58, n. 4, p. 414-422, 2005.

MARKETING SCIENCE INSTITUTE. 1998-2000 research priorities: a guide to MSI research programs and procedures. Cambridge, MA: MSI, 1998.

MOYSÉS FILHO, J. E. et al. Entre a teoria e a prática no desenvolvimento e lançamento novos produtos em PMEs: o caso de uma nova sobremesa para o mercado food service. In: CONGRESSO LATINO-AMERICANO DE VAREJO, 1., 2008, São Paulo. Anais... São Paulo: FGV, 2008. 1 CD-ROM.

NARVER, J. C.; SLATER, S. F. The effect of a market orientation on business profitability. Journal of Marketing, New York, v. 54, n. 4, p. 20-35, 1990.

NUNNALLY, J. C. Psychometric theory. $2^{\text {nd }}$ ed. New York: McGraw-Hill, 1978.

O’SULLIVAN, D.; ABELA, A. V. Marketing performance measurement ability and firm performance. Journal of Marketing, New York, v. 71, n. 2, p. 72-93, 2007.

PERIN, M. G.; SAMPAIO, C. H. Orientação para o mercado, porte empresarial e performance: um estudo longitudinal no setor eletro eletrônico do Brasil. In: ENCONTRO DA ASSOCIAÇÃO NACIONAL DE PÓS-GRADUAÇÃO E PESQUISA EM ADMINISTRAÇÃ̃O, 27., 2003, Atibaia. Anais... Rio de Janeiro: ANPAD, 2003. 1 CD-ROM.

RIBEIRO, Á. H. P. et al. Proposta de uma nova escala para medir competência em Marketing. Revista de Administração e Inovação, São Paulo, v. 3, n. 1, p. 5-20, 2006.

RIES, A.; TROUT, J. Marketing warfare. New York: McGraw Hill, 1986.

ROBINSON, W. T.; FOMELL, C. Market pioneering and sustainable market share advantage. Cambridge: Strategic Planning Institute, 1986.

RUST, R. T. et al. Measuring marketing productivity: current knowledge and future directions. Journal of Marketing, New York, v. 68, n. 4, p. 76-90, 2004a.

RUST, R. T.; LEMON, K. N.; ZEITHAML, V. A. Return on marketing: using customer equity to focus marketing strategy. Journal of Marketing, v. 68, n. 1, p. 109-127, 2004b. 
SHETH, J. N.; SISODIA, R. S. Feeling the heat - part I, Marketing Management, Chicago, v. 4, n. 2, p. 9-23, 1995a.

SHETH, J. N.; SISODIA, R. S. Feeling the heat - part II, Marketing Management, Chicago, v. 4, n. 3, p. 19-33, 1995b.

SLATER, S. F.; NARVER, J. C. Market orientation and the learning organization. Journal of Marketing, New York, v. 59, n. 3, p. 63-74, 1995.

SONG, X. M.; PARRY, M. E. A cross-national comparative study of new product development processes: Japan and United State. Journal of Marketing, New York, v. 61, n. 2, p. 1-18, 1997.

SZYMANSKI, D. M.; BHARADWAJ, S. G.; VARADARAJAN, P. R. An analysis of the market share-profitability relationship. Journal of Marketing, New York, v. 57, n. 3, p. 1-18, 1993.

TAVARES, M. C.; PINTO, V. B.; AFONSO, T. Orientação para o mercado e novos produtos sob o enfoque do sistema de consumo. In: ENCONTRO DE MARKETING, 2., 2006, Rio de Janeiro. Anais... Rio de Janeiro: ANPAD, 2006. 1 CD-ROM.

STRATEGIC PLANNING INSTITUTE. Profit impact of market strategy: overview. Disponível em: <http://pimsonline.com/about_pims_db.htm>. Acesso em: 12 out. 2009.

URDAN, A. T.; OSAKU, W. A. A. Determinantes do sucesso de novos produtos: um estudo de empresas estrangeiras no Brasil. In: ENCONTRO DA ASSOCIAÇÃO NACIONAL DE PÓS-GRADUAÇÃO E PESQUISA EM ADMINISTRAÇÃO, 29., 2005, Brasília, DF. Anais... Rio de Janeiro: ANPAD, 2005. 1 CD-ROM.

VORHIES, D. W.; HARKER, M. The capabilities and performance advantages of marketdriven firms. European Journal of Marketing, West Yorkshire, v. 33, n. 11/12, p. 11711202, 1999.

VORHIES, D. W.; HARKER, M. The capabilities and performance advantages of marketdriven firms: an empirical investigation. Australian Journal of Management, Sydney, v. 25, n. 2, p. 145-173, 2000.

VORHIES, D. W.; MORGAN, N. A. Benchmarking marketing capabilities for sustainable competitive advantage. Journal of Marketing, New York, v. 69, n. 1, p. 80-94, 2005.

WHEELWRIGHT, S.; CLARK, K. Accelerating the design-build-test cycle for effective product development. International Marketing Review, London, v. 11, n. 1, p. 32-46, 1994.

APÊNDICE

Apêndice - A - Variáveis da pesquisa de campo

\begin{tabular}{|l|l|}
\hline Variáveis & \\
\hline & Product investment Variables (Measures) \\
\hline Produto1 & Research and development (money/employees] \\
\hline Produto2 & Patents (number issued and pending) \\
\hline Produto3 & Production capacity (value/employees/units) \\
\hline
\end{tabular}


AMBIÇÃO ESTRATÉGICA DE MARKETING: O CASO DO SEGMENTO QUÍMICOFARMACÊUTICO.

\begin{tabular}{|l|l|}
\hline Produto4 & Assortment (number offered) \\
\hline Produto5 & Plant inventories (value/units) \\
\hline Produto6 & Product performance (technical/perceptual) \\
\hline Produto7 & Trademarks (value/number of brands) \\
\hline Produto8 & Warranties (value/terms) \\
\hline & Promotional investment Variables (Measures) \\
\hline Propag1 & Media (money/number of messages) \\
\hline Propag2 & Salespersons (money/number/calls) \\
\hline Propag3 & Promotion (money/number) \\
\hline Propag4 & Publicity (number of messages) \\
\hline Propag5 & Positioning (metric distance) \\
\hline Propag6 & Message (impact) \\
\hline Propag7 & Production (value) \\
\hline & Piace Investment Variables (Measures) \\
\hline Distrib1 & Retail Outiets (number) \\
\hline Distrib2 & Retail salespersons (number) \\
\hline Distrib3 & Field inventories (value/units) \\
\hline Distrib4 & Selling space (square feet) \\
\hline Distrib5 & Shelf space (linear feet/facings) \\
\hline Distrib6 & Hours of business \\
\hline Distrib7 & Special measures (e.g., flights per city pair) \\
\hline Distrib8 & Trade support (dollar margins x volume) \\
\hline & Price Investment Variables (Measures) \\
\hline Pol_Coml1 & Primary demand elasticity \\
\hline Pol_Coml2 & Price Relative (1 - P/P*) \\
\hline Pol_Coml3 & Rebates/discounts (money) \\
\hline Pol_Coml4 & Trade margins (percent) \\
\hline Pol_Coml5 & Terms of sale (time/interest) \\
\hline Pol_Coml6 & Refund policy (recovery value) \\
\hline Pol_Coml7 & Transaction cost (money) \\
\hline & \\
\hline & \\
\hline &
\end{tabular}

Fonte: Cook Junior (1983) 